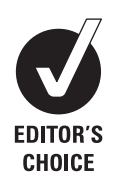

CHOICE

\title{
Weight loss in obese people has structure-modifying effects on medial but not on lateral knee articular cartilage
}

\author{
A Anandacoomarasamy, ${ }^{1-3}$ S Leibman, ${ }^{3,4}$ G Smith, ${ }^{3,4}$ I Caterson, ${ }^{5}$ B Giuffre, ${ }^{3,6}$ \\ M Fransen, ${ }^{7}$ P N Sambrook, ${ }^{1,3}$ L March $^{1,3}$
}

\begin{abstract}
- Additional data are published online only. To view the files please visit the journal online (http://ard.bmi.com content/71/1.toc)
\end{abstract}

${ }^{1}$ Institute of Bone and Joint Research, Kolling Institute, Royal North Shore Hospital, University of Sydney, Sydney, New South Wales, Australia 2Rheumatology, Concord Hospital, Sydney, Australia ${ }^{3}$ University of Sydney, Sydney, New South Wales, Australia ${ }^{4}$ Royal North Shore Hospital, Sydney, Department of Surgery, New South Wales, Australia ${ }^{5}$ Human Nutrition, University of Sydney, Sydney, New South Wales, Australia

${ }^{6}$ Radiology, Royal North Shore Hospital, Sydney, New South Wales, Australia

${ }^{7}$ Clinical and Rehabilitation Sciences Research Group, University of Sydney, Sydney, New South Wales, Australia

\section{Correspondence to}

Professor L March, Department of Rheumatology, Royal North Shore Hospital, University of Sydney, Building 35, Level 4, St Leonards, Sydney, NSW 2065, Australia;

lynmar@med.usyd.edu.au

Accepted 14 August 2011

\section{ABSTRACT}

Background Obesity is an important risk factor for knee osteoarthritis (OA), Weight loss can reduce the symptoms of knee OA. No prospective studies assessing the impact of weight loss on knee cartilage structure and composition have been performed.

Objectives To assess the impact of weight loss on knee cartilage thickness and composition.

Methods 111 obese adults were recruited from either laparoscopic adjustable gastric banding or exercise and diet weight loss programmes from two tertiary centres. MRI was performed at baseline and 12-month follow-up to assess cartilage thickness. 78 eligible subjects also underwent delayed gadolinium-enhanced MRI of cartilage (dGEMRIC), an estimate of proteoglycan content. The associations between cartilage outcomes (cartilage thickness and dGEMRIC index) and weight loss were adjusted for age, gender, body mass index (BMI) and presence of clinical knee $0 A$.

Results Mean age was $51.7 \pm 11.8$ years and mean BMl was $36.6 \pm 5.8 \mathrm{~kg} / \mathrm{m}^{2} ; 32 \%$ had clinical knee 0 A. Mean weight loss was $9.3 \pm 11.9 \%$. Percentage weight loss was negatively associated with cartilage thickness loss in the medial femoral compartment in multiple regression analysis $\left(\beta=0.006, r^{2}=0.19, p=0.029\right)$. This association was not detected in the lateral compartment $\left(r^{2}=0.12, p=0.745\right)$. Percentage weight loss was associated with an increase in medial dGEMRIC in multiple regression analysis $(\beta=3.9$, $r^{2}=0.26 ; p=0.008$ ) but not the lateral compartment $\left(r^{2}=0.14, p=0.34\right)$. For every $10 \%$ weight loss there was a gain in the medial dGEMRIC index of $39 \mathrm{~ms}\left(\mathrm{r}^{2}=0.28\right.$; $p=0.014)$. The lowest weight loss cut-off associated with reduced medial femoral cartilage thickness loss and improved medial dGEMRIC index was 7\%.

Conclusions Weight loss is associated with improvements in the quality (increased proteoglycan content) and quantity (reduced cartilage thickness losses) of medial articular cartilage. This was not observed in the lateral compartment. This could ultimately lead to a reduced need for total joint replacements and is thus a finding with important public health implications.

\section{INTRODUCTION}

Obesity represents a major public health problem. The WHO estimates that more than one billion people are overweight and, of these, 300 million are obese. ${ }^{1}$ In addition, the levels of extreme obesity (obesity grade 3 , body mass index (BMI) $\geq 40$ $\mathrm{kg} / \mathrm{m} 2$ ) are also escalating. ${ }^{2}$
Osteoarthritis $(\mathrm{OA})$ is the most common form of arthritis and the leading cause of chronic disability among older people. Obesity is a significant risk factor for the incidence of knee OA, but the effects on disease progression are less consistent. ${ }^{3-5}$ In an analysis of the direct costs of obesity it was estimated that the cost of OA in the USA (US\$5.3 billion) was second only to the cost of diabetes in obesity-associated conditions. ${ }^{6} \mathrm{OA}$ has a significant negative impact on most economies-for example, in the UK economy, OA has a total cost estimated to be equivalent to $1 \%$ of Gross National Product per year. ${ }^{7}$ Obesity-related $\mathrm{OA}$ is estimated to be responsible for at least $10 \%$ of this cost. $^{8}$

OA affects articular cartilage and other structures such as subchondral bone and meniscus. Loss of articular cartilage is a marker of OA severity. ${ }^{9}$ The main function of articular cartilage is to permit frictionless and pain-free movement of the joint. ${ }^{10}$ Articular cartilage consists of a large extracellular matrix composed of water and proteoglycans entrapped within a collagenous framework. Proteoglycans are made up of glycosaminoglycans (GAGs) attached to a backbone of hyaluronic acid. ${ }^{11}$ Proteoglycans provide the cartilage with compressive stiffness.

Quantitative cartilage assessment using MRI allows the measurement of important cartilage structural features such as thickness and volume. ${ }^{12}$ In comparison with radiography, MRI detects morphological changes in cartilage at a much earlier stage of the disease- that is, it allows the detection of pre-radiographic OA. ${ }^{12}$

Delayed gadolinium-enhanced MRI of cartilage (dGEMRIC), a cartilage compositional measure, is used to assess the relative distribution of GAG in cartilage non-invasively. ${ }^{13}$ GAGs are negatively charged due to abundant carboxyl and sulfate groups that are ionised at physiological $\mathrm{pH}$. The technique uses a negatively charged contrast agent, gadopentate dimeglumine (Gd-DTPA ${ }^{2-}$; Magnevist; Berlex Laboratories, Wayne, New Jersey, USA) which, when given time to penetrate cartilage tissue, distributes within the cartilage matrix in an inverse relationship to the concentration of negatively charged GAG. ${ }^{111415}$ The concentration of Gd-DTPA will therefore be relatively low in normal (GAG-abundant) cartilage and relatively high in degraded cartilage (GAG loss). This allows calculation of the dGEMRIC index, with a low GAG content resulting in a low dGEMRIC index and a high GAG content yielding a high dGEMRIC 
index. ${ }^{11}$ The associations between BMI and the dGEMRIC index in cross-sectional analysis have been inconsistent. ${ }^{11} 16$

Weight loss has been shown to reduce knee pain and to improve knee stiffness, function and disability. ${ }^{17} 18$ No studies to date have assessed the effects of weight loss on MRI cartilage structural outcomes. The aim of the current study was to evaluate the effect of weight loss on cartilage thickness and GAG content in a cohort of obese people participating in weight loss programmes.

\section{METHODS}

\section{Study population}

In this observational prospective cohort study, patients were recruited from two weight loss (non-surgical or surgical) programmes in which patients had voluntarily enrolled themselves. All subjects were obese $\left(\mathrm{BMI}>30 \mathrm{~kg} / \mathrm{m}^{2}\right)$, with most being obesity grade 2 or higher (ie, BMI $>35 \mathrm{~kg} / \mathrm{m}^{2}$ ). The non-surgical programme (dietary modification and exercise) was conducted at the Metabolism and Obesity Services Clinic of the Royal Prince Alfred Hospital, Sydney. The surgical group underwent laparoscopic adjustable gastric banding by one of two experienced surgeons at Royal North Shore Hospital, Sydney. All patients were screened during their initial visit to the respective centres and offered the opportunity to participate in this longitudinal observational study.

Apart from the usual MRI exclusion criteria, clinical exclusion criteria included inflammatory arthritis or psychiatric illness. dGEMRIC exclusion criteria include glomerular filtration rate $<60 \mathrm{mmol} / \mathrm{l}$, pregnancy or breast feeding. The American College of Rheumatology clinical classification criteria were used to define knee OA. ${ }^{19}$ This requires the presence of knee pain on most days of the last month and at least three of the following: age $>50$ years, morning stiffness $<30 \mathrm{~min}$, crepitus, bony tenderness, bony enlargement and no palpable warmth.

\section{Assessments}

One hundred and eleven subjects were recruited. Subjects were assessed at recruitment (baseline, prior to commencement of the weight loss programme) and again 12 months later.

\section{Clinical assessment}

The weight loss percentage was calculated as: (weight loss/baseline weight) $\times 100 \%$. Weight loss is defined as any loss in weight and weight gain is defined as any gain in weight. Knee range of motion and alignment were assessed in all subjects. Measurement methods have been described in detail elsewhere. ${ }^{20-22}$

\section{MRI assessment}

\section{Cartilage thickness}

Eligible subjects underwent baseline MRI of the symptomatic or dominant asymptomatic knee. Sagittal MRI images were obtained on a 3T scanner (Magnetom Trio; Siemens, Erlangen, Germany) as previously described. ${ }^{23}$ Cartilage segmentation was performed by a single trained reader (AA) blinded to all clinical data including age, presence of knee symptoms and degree of weight loss. The segmentations were used to analyse cartilage thickness (weight-bearing femoral cartilage and all of the tibial cartilage) using proprietary software (Chondrometrics, Ainring, Germany), based on previously described and validated methods. ${ }^{24}$

\section{Knee dGEMRIC assessment}

A standard dGEMRIC protocol was applied. dGEMRIC images were obtained in the sagittal planes as previously described. ${ }^{11}$ Double dose $(0.2 \mathrm{mM} / \mathrm{kg})$ GdDTPA ${ }^{2-}$ was administered $90 \mathrm{~min}$ prior to imaging at baseline and 12 months. ${ }^{11}$ Subjects were required to walk for 15 min after injection. T1Gd maps were generated to calculate the dGEMRIC index with a pixel-by-pixel three-parameter T1 fit using Matlab software (The MathWorks, Natick, Massachusetts, USA). The mean medial and lateral dGEMRIC indices were obtained. The dGEMRIC indices were calculated after manual segmentation for four regions of interest (ROIs), two each from the medial and lateral sagittal sections, to yield the mean dGEMRIC index for an individual ROI, as well as averaged across sagittal views to obtain the medial and lateral dGEMRIC index. Full-thickness ROIs in the sagittal plane consisted of weight-bearing femoral cartilage and all of the tibial cartilage. All images were read by a single trained observer (AA) blinded to all clinical data. The BMI correction equation was also applied for completion. However, this may not be applicable for 3T MRI imaging. ${ }^{25}$

Intraobserver reliability was measured using intraclass correlation coefficients (ICCs) after repeat mapping 1 week apart for 20 subjects for both cartilage thickness and dGEMRIC (ICC $>0.91$ for each $\mathrm{ROI}$ ).

\section{Statistical analysis}

The mean change over 12 months and standardised response means (mean change/SD of change) were calculated. Spearman correlation analysis was used to analyse the association between change in dGEMRIC indices, cartilage thickness and percentage weight loss. The Mann-Whitney test was used for categorical variables. Linear regression was used to examine univariate associations between percentage weight loss (covariate) and MRI cartilage outcomes (dependent variable). Multiple regression analysis was adjusted for age, gender, baseline BMI and presence of clinical knee OA. All statistical analyses were carried out using SPSS standard version 16.0 (SPSS, Chicago, Illinois, USA). A p value $<0.05$ was regarded as statistically significant.

\section{RESULTS \\ Cartilage thickness}

One hundred and eleven subjects underwent baseline MRI assessment of cartilage thickness. Seventy-eight patients $(70 \%)$ completed MRI follow-up for cartilage assessment at 12 months. The reasons for loss to follow-up are given in the online supplement (see figure S1). There were no significant demographic differences at baseline between the group that underwent MRI follow-up ( $n=78$ ) at 12 months and the group that did not. The baseline characteristics of the cohort are shown in table 1 . The group that underwent surgery for weight loss achieved greater weight loss than the non-surgical group $(17.5 \%$ and $2.3 \%$, respectively). There were no significant differences in mean cartilage thickness or dGEMRIC values or other demographic values at baseline between the two groups.

\section{Change in cartilage thickness over 12 months}

The mean change in compartment-specific cartilage thickness over 12 months is shown in table 2. Mean change in cartilage thickness was lower in subjects who lost weight, which was significant at the medial tibia (MT) and central medial femur (cMF). This was not observed in the lateral compartment. There was no difference in loss of cartilage thickness between subjects with and without clinical knee $O A$.

\section{Change in cartilage thickness and weight loss}

A higher percentage weight loss was associated with reduced loss of cartilage thickness in the medial femoral compartment in univariate (table 3 ) and multiple regression analysis adjusted 
Table 1 Baseline characteristics of the obese cohort

\begin{tabular}{lll}
\hline Characteristic & $\begin{array}{l}\text { Underwent cartilage } \\
\text { thickness assessment }\end{array}$ & $\begin{array}{l}\text { Underwent dGEMRIC } \\
\text { assessment }\end{array}$ \\
\hline Number of subjects & 78 & 55 \\
Age (years) & $51.7 \pm 11.8$ & $50.9 \pm 12.4$ \\
Female (\%) & $75 \%$ & $67 \%$ \\
BMI (kg/m2) & $39.9 \pm 5.8$ & $39.6 \pm 5.2$ \\
Knee osteoarthritis (ACR) (\%) & $32 \%$ & $26 \%$ \\
Surgery for weight loss (\%) & $36 \%$ & $44 \%$ \\
Weight loss (12 months) (\%) & $9.3 \pm 11.9$ & $9.1 \pm 10.0$ \\
Medial tibial cartilage thickness (mm) & $1.40 \pm 0.21$ & $\mathrm{NA}$ \\
Medial femoral cartilage thickness (mm) & $1.75 \pm 0.34$ & $\mathrm{NA}$ \\
Lateral tibial cartilage thickness (mm) & $1.93 \pm 0.32$ & $\mathrm{NA}$ \\
Lateral femoral cartilage thickness (mm) & $1.95 \pm 0.36$ & $\mathrm{NA}$ \\
Medial dGEMRIC index (ms) & $\mathrm{NA}$ & $545 \pm 84$ \\
Lateral dGEMRIC index (ms) & $\mathrm{NA}$ & $539 \pm 91$ \\
\hline
\end{tabular}

Data shown are mean $\pm S D$ or percentage.

ACR, American College of Rheumatology; BMI, body mass index; dGEMRIC, delayed gadolinium-enhanced MRI of cartilage.

Table 2 Mean \pm SD change in cartilage thickness $(\mathrm{mm})$

\begin{tabular}{|c|c|c|c|c|c|c|c|c|c|}
\hline & \multicolumn{3}{|c|}{ Chanwge $(\mathrm{mm})^{*}$} & \multicolumn{3}{|c|}{ Annual change $(\%) \dagger$} & \multicolumn{3}{|c|}{ Cartilage thickness (SRM¥) } \\
\hline & All $(n=78)$ & $\begin{array}{l}\text { Weight loss } \\
(n=59)\end{array}$ & $\begin{array}{l}\text { Weight gain/no } \\
\text { change }(n=19)\end{array}$ & All $(n=78)$ & $\begin{array}{l}\text { Weight loss } \\
(n=59)\end{array}$ & $\begin{array}{l}\text { Weight gain/no } \\
\text { change }(n=19)\end{array}$ & All $(n=78)$ & $\begin{array}{l}\text { Weight loss } \\
(n=59)\end{array}$ & $\begin{array}{l}\text { Weight gain/no } \\
\text { change }(n=19)\end{array}$ \\
\hline MT & $-0.02 \pm 0.1$ & $-0.006 \pm 0.1$ & $-0.06 \pm 0.2$ & $-1.3 \pm 9.5$ & $-0.5 \pm 8.4$ & $-3.9 \pm 12.1$ & -0.15 & -0.06 & -0.35 \\
\hline cMF & $-0.004 \pm 0.2$ & $0.03 \pm 0.2$ & $-0.07 \pm 0.2$ & $1.0 \pm 17.5$ & $2.6 \pm 18.9$ & $-3.6 \pm 11.4$ & -0.02 & 0.12 & -0.32 \\
\hline LT & $-0.006 \pm 0.1$ & $-0.006 \pm 0.1$ & $-0.007 \pm 0.2$ & $0.1 \pm 8.1$ & $0.02 \pm 7.5$ & $0.4 \pm 10.0$ & -0.05 & -0.05 & -0.04 \\
\hline $\mathrm{cLF}$ & $-0.07 \pm 0.2$ & $-0.08 \pm 0.2$ & $-0.05 \pm 0.2$ & $-3.6 \pm 9.3$ & $-4.1 \pm 9.2$ & $-2.1 \pm 9.6$ & -0.40 & -0.45 & -0.26 \\
\hline
\end{tabular}

Weight loss, any loss in weight; no change, no change in weight; weight gain, any gain in weight.

*Difference in cartilage thickness between follow-up and baseline.

$\dagger$ Annual rate of change $(\% /$ year $)=$ (change in cartilage thickness/baseline cartilage thickness $) \times 100 \%$.

$\ddagger$ Standardised response mean (mean/SD of change).

cLF, central lateral femur ${ }^{58}$; cMF, central medial femur; LT, lateral tibia; MT, medial tibia.

Table 3 Change in cartilage thickness $(\mathrm{mm})$ and weight loss: univariate analysis

\begin{tabular}{|c|c|c|c|c|}
\hline \multirow[b]{2}{*}{ Region } & \multicolumn{4}{|c|}{ Univariate } \\
\hline & $\bar{r}$ & p Value & $\beta$ & $95 \% \mathrm{Cl}$ \\
\hline MT & -0.22 & 0.058 & 0.003 & 0.000 to 0.006 \\
\hline $\mathrm{cMF}$ & -0.30 & 0.009 & 0.007 & 0.002 to 0.012 \\
\hline LT & 0.11 & 0.383 & -0.001 & -0.005 to 0.003 \\
\hline $\mathrm{cLF}$ & 0.05 & 0.677 & 0.000 & -0.005 to 0.004 \\
\hline
\end{tabular}

cLF, central lateral femur; cMF, central medial femur; LT, lateral tibia; MT, medial tibia.

for age, baseline BMI, gender and clinical knee $\mathrm{OA}(\beta=0.006$, $\left.\mathrm{r}^{2}=0.19, \mathrm{p}=0.029\right)$. A similar association did not reach statistical significance in the MT compartment. The degree of cartilage loss in the MT ( $p=0.181)$ and medial femoral $(p=0.06)$ compartment was less with increasing quartiles of weight loss percentage but this model did not reach statistical significance. Lateral cartilage thickness loss was not associated with percentage weight loss in univariate or multivariable analysis.

The mean percentage weight loss for the cohort of $9 \%$ was used as a cut-off point for weight loss to generate two groups. There was a significant difference in cartilage thickness loss in the medial femoral compartment between the two groups, with the group losing more weight having a lower cartilage thickness loss $(p=0.02)$. In order to provide a potential clinically relevant weight loss target, a series of sensitivity analyses were run to determine the lowest percentage of weight loss that remained significantly associated with cartilage outcomes after adjusting for the confounders. In this cohort, weight loss of at least $7 \%$ was still significantly associated with reduced cartilage thickness loss while adjusting for age, gender, baseline BMI and the presence of clinical OA.

Improved knee range of motion was associated with reduced loss of cartilage thickness in the medial compartment at 12 months (MT: $r=0.20 ; p=0.079$; $c M F: r=0.37 ; p=0.001$ ).

\section{dGEMRIC}

Of the 111 subjects who underwent baseline MRI, 78 subjects underwent baseline dGEMRIC assessment. The reasons for not undergoing dGEMRIC assessment are shown in the online supplement. Fifty-five patients (71\%) completed follow-up dGEMRIC assessment at 12 months. The reasons for loss to follow-up are shown in the flow chart in the online supplement. There were no significant baseline differences between the group that underwent dGEMRIC assessment at 12 months and the group that did not. The baseline characteristics of the cohort are shown in table 1.

\section{Change in dGEMRIC over 12 months}

One subject with severe cartilage thinning was excluded from dGEMRIC assessment as previously described. ${ }^{11}$ The overall change in dGEMRIC indices is shown in table 4. Subjects who lost weight had a higher mean gain in medial and lateral dGEMRIC indices. However, these differences did not reach statistical significance.

\section{Change in dGEMRIC index and weight loss}

An improved medial dGEMRIC index correlated positively with percentage weight loss in univariate analysis 
Table 4 Mean \pm SD dGEMRIC index (ms)

\begin{tabular}{lccc}
\hline & & \multicolumn{2}{l}{ Cohort (n=54) } \\
\cline { 3 - 4 } Region of interest & Change & $\begin{array}{l}\text { Weight loss } \\
(\mathbf{n = 4 1 )}\end{array}$ & $\begin{array}{l}\text { Weight gain/no } \\
\text { change }(\mathbf{n}=\mathbf{1 3})\end{array}$ \\
\hline Medial tibial & $23 \pm 125.7$ & $25 \pm 128$ & $-4 \pm 123$ \\
Medial femoral & $2 \pm 123.0$ & $3 \pm 138$ & $-5 \pm 60$ \\
Medial dGEMRIC index & $13 \pm 106$ & $17 \pm 113$ & $-3 \pm 78$ \\
Medial dGEMRIC index & $3 \pm 103$ & $4 \pm 110$ & $-21 \pm 65$ \\
$\quad$ (BMI corrected) & $23 \pm 135$ & $24 \pm 138$ & $19 \pm 128$ \\
Lateral tibial & $23 \pm 107$ & $29 \pm 114$ & $-2 \pm 89$ \\
Lateral femoral & $23 \pm 107$ & $27 \pm 114$ & $8 \pm 83$ \\
Lateral dGEMRIC index & $11 \pm 106$ & $12 \pm 113$ & $2 \pm 82$ \\
Lateral dGEMRIC index & & & \\
$\quad$ (BMI corrected) & & & \\
\hline
\end{tabular}

$\mathrm{BMI}$, body mass index; dGEMRIC, delayed gadolinium-enhanced MRI of cartilage.

( $\mathrm{r}=0.35 ; \mathrm{p}=0.011$; figure 1 ). In multiple regression analysis adjusting for age, gender, baseline BMI and presence of clinical knee $\mathrm{OA}$, an improved medial dGEMRIC index correlated positively with percentage weight loss $\left(\beta=3.9, \mathrm{r}^{2}=0.26 ; \mathrm{p}=0.008\right)$. The degree of improvement in the mean medial dGEMRIC index improved incrementally with increasing quartiles of weight loss percentage, but this model did not reach statistical significance $(p=0.07)$. The change in the lateral dGEMRIC index was not associated with percentage weight loss in univariate analysis or in multivariable analysis (adjusting for age, gender, baseline BMI and presence of clinical knee $\mathrm{OA}$ ).

The mean percentage weight loss for the cohort of $9 \%$ was used as a cut-off point for weight loss to generate two groups (table 5). The mean difference in medial dGEMRIC index change between the two groups was $61 \mathrm{~ms}$ ( $95 \% \mathrm{CI}-118$ to -5 ; $\mathrm{p}=0.034$; effect size 0.6 ). Overall, $7 \%$ weight loss was the lowest cut-off associated with a significant difference in medial dGEMRIC index in this cohort. Figure 2 shows the changes seen in the medial dGEMRIC index in a study participant with weight loss.

Improved knee range of motion correlated with improved medial dGEMRIC index in univariate analysis $(\mathrm{r}=0.28$; $p=0.046$ ). The multivariable model that included age, gender, baseline BMI, knee OA and change in knee range of motion accounted for $28 \%$ of the variance in medial dGEMRIC with

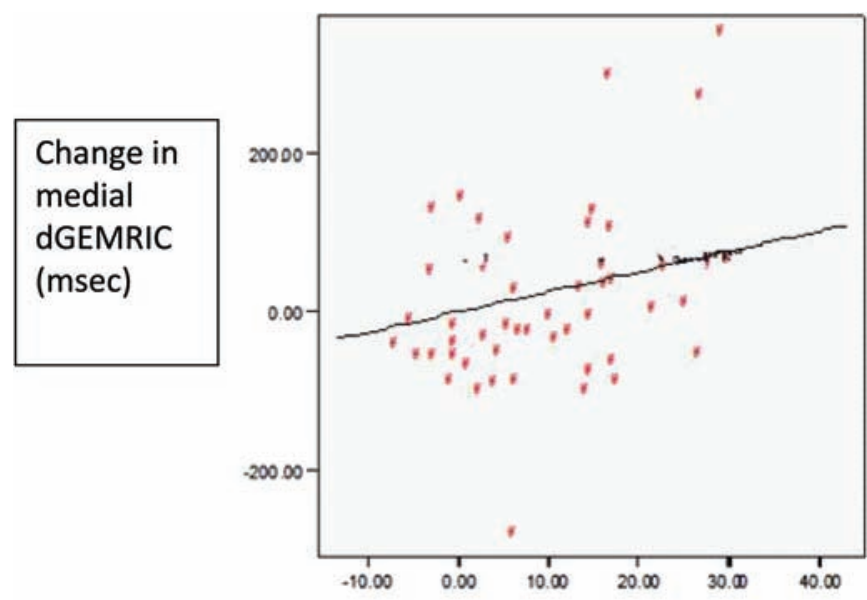

Change in weight loss percent (\%)

Figure 1 Correlation between change in medial delayed gadoliniumenhanced MRI of cartilage (dGEMRIC) and weight loss percentage.
Table 5 Mean \pm SD change in medial dGEMRIC index (ms) according to $9 \%$ weight loss cut-off point

\begin{tabular}{lll}
\hline Region & $\begin{array}{l}\text { Group 1 ( } \mathbf{n}=\mathbf{2 5}) \\
\text { (weight loss } \geq \mathbf{9 \%})\end{array}$ & $\begin{array}{l}\text { Group } \mathbf{2} \text { ( } \mathbf{n}=\mathbf{2 8}) \text { (gained weight, } \\
\text { no change or weight loss }<\mathbf{9 \%}\end{array}$ \\
\hline Medial dGEMRIC & $45 \pm 116$ & $-16 \pm 88$ \\
$\begin{array}{l}\text { Medial dGEMRIC } \\
\text { (BMI corrected) }\end{array}$ & $22 \pm 114$ & $-18 \pm 90$ \\
\hline
\end{tabular}

BMI, body mass index; dGEMRIC, delayed gadolinium-enhanced MRI of cartilage.

percentage weight loss $\left(r^{2}=0.28 ; p=0.014\right)$. In this model, for every $10 \%$ weight loss there was a gain in the medial dGEMRIC index of $39 \mathrm{~ms}$.

\section{Change in dGEMRIC index and cartilage thickness}

Reduced loss of MT cartilage thickness was positively associated with improved MT dGEMRIC index $(\mathrm{r}=0.35, \mathrm{p}=0.011)$. These associations were not significant in multivariable regression analysis.

\section{DISCUSSION}

This is the first prospective study to evaluate the impact of weight loss on knee articular cartilage structure and quality. To the best of our knowledge, there are no other published studies assessing the impact of weight loss intervention on knee articular cartilage. We demonstrated that weight loss is associated with reduced cartilage thickness losses in the medial femoral compartment as well as improved medial dGEMRIC index (ie, proteoglycan content). No association was identified between weight loss and change in cartilage thickness or dGEMRIC index in the lateral compartment. Improved knee range of motion was independently associated with reduced loss of cartilage thickness. The degree of weight loss observed in the surgical and non-surgical groups is similar to published reports. ${ }^{26-28}$

There have been several published reports of the annual rate of change of cartilage thickness. ${ }^{29-31}$ Recent early data from the osteoarthritis initiative (OAI), a multicentre observational study evaluating risk factors for the incidence and progression of symptomatic knee OA, demonstrated a modest but significant loss of tibio-femoral cartilage thickness over 1 year $^{2932}$ in people with frequent knee symptoms and radiographic knee OA. The effect sizes (standardised response mean) of cartilage thickness loss in the OAI cohort varied within knee joint compartments (MT -0.16; cMF -0.30; lateral tibia -0.23; cLF-0.02). These cartilage thickness losses are larger than those of our weight loss subgroup in the medial compartment (MT -0.06; cMF 0.12). In addition, the OAI subcohort had severe knee OA whereas our cohort consisted largely of subjects without clinical knee OA, albeit at higher risk of knee OA due to morbid obesity. Notably, our study involved effective weight loss interventions, and we hypothesised lower effect sizes, indicating reduced cartilage loss. Interestingly, however, the patients who gained weight demonstrated effect sizes similar to the OAI cohort with knee OA (MT -0.35; cMF -0.33). The difference in mean cartilage thickness findings in the cLF between recent studies and this cohort could partly be related to the large range of weight loss shown in this relatively small cohort compared with the OAI cohorts. Increasing levels of obesity can impair the quality of the MRI images obtained and hence alter measurements. However, a surface coil is required in the research setting and, in our study, ${ }^{33}$ very obese patients with a thigh too large for the coil were excluded. This acquisition methodology limits variability in the obtained images. 


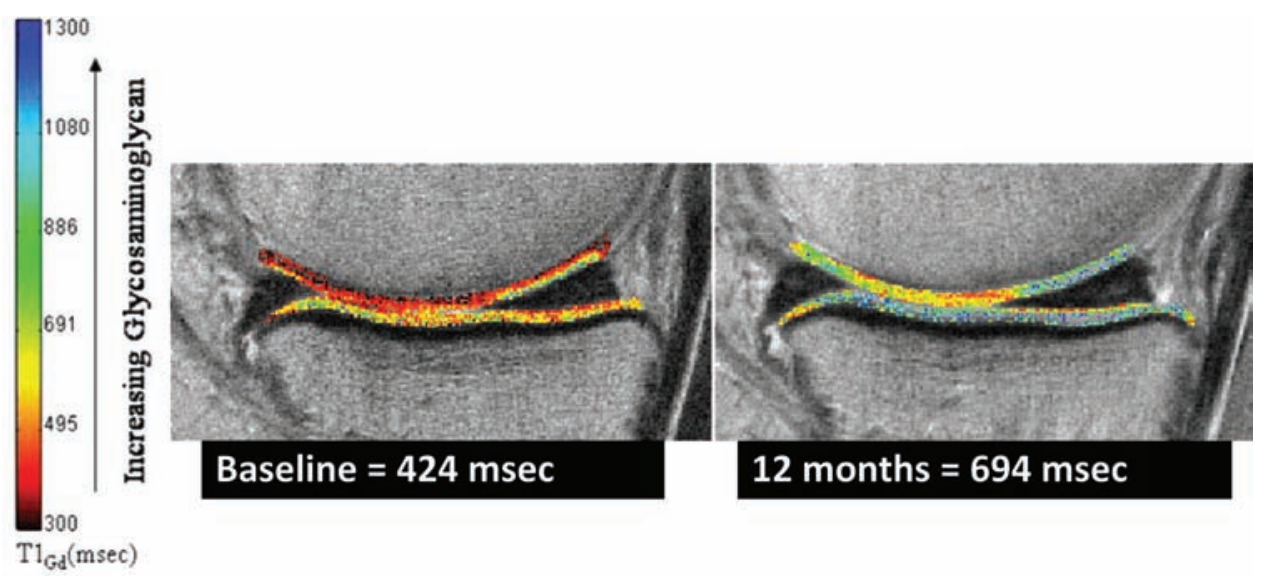

Figure 2 Change in medial delayed gadolinium-enhanced MRI of cartilage (dGEMRIC) at 12 months (patient with weight loss $43 \mathrm{~kg}$; change in body mass index 13 units).

Importantly, weight loss was associated with lower cartilage thickness losses in the medial compartment and after adjustment for potential confounders. Similarly, the effect of weight loss on the medial dGEMRIC index remained significant after adjustment for potential confounders. We did not observe similar associations between lateral cartilage thickness loss or the dGEMRIC index with weight loss. The differential change in the medial compared with the lateral compartment is not unexpected as previous studies have found that the medial compartment generally exhibits higher rates of cartilage loss in people with knee OA. ${ }^{31} 3435$ This finding is likely to be attributable to the greater proportion of ground reaction forces borne by the medial tibiofemoral compartment, even in normally aligned knees. ${ }^{36}$ A previous study also demonstrated a lower medial dGEMRIC index in varus-aligned knees and a lower lateral dGEMRIC index in valgus-aligned knees. ${ }^{22}$ While this remains speculative, the potential for improvement for most obese people with knee OA undergoing weight loss is possibly greater in the medial compartment. ${ }^{31}$ The lack of association in the lateral compartment in this cohort is not explained. In this cohort the baseline femoral cartilage was thicker than the tibial cartilage, contrary to normal values for OA cohorts. This may be a reflection of a morbidly obese population and needs to be assessed further in other obese cohorts. Further research is also required to determine the mechanisms of cartilage change mediated by weight loss, both biomechanical and biochemical.

The quantification techniques used have previously been validated. ${ }^{37-39}$ Cartilage loss as measured by MRI over relatively short periods ( 1 or 2 years) has been shown to be associated with cartilage loss over longer periods (4.5 years). ${ }^{40}$ In addition, cartilage loss has been shown to correlate with knee arthroplasty, making it a clinically important surrogate end point. ${ }^{41} 42$

The dGEMRIC index has also been shown to be a clinically relevant measure of cartilage integrity. A recent study indicated that low dGEMRIC may predict future radiographic knee OA. ${ }^{43}$ The dGEMRIC index has been shown to be low in individuals with moderate to severe radiographic knee OA, ${ }^{15}$ after cruciate ligament injury ${ }^{44} 45$ and in diseased knee compartments on arthroscopy. ${ }^{46}$ In addition, a range of dGEMRIC values has been observed in radiographically comparable compartments demonstrating biochemical differentiation of disease. ${ }^{22}$ In our cohort we observed different dGEMRIC indices in patients with similar cartilage thickness measurements. Improvements in dGEMRIC index have also been observed in patients with hip dysplasia undergoing pelvic osteotomy. ${ }^{47}$ These findings suggest that early cartilage degradation may be reversible. This has great clinical relevance as it indicates an opportunity for intervention or change before irreversible change in cartilage morphometry occurs. The BMI dose correction equation was also applied but this did not change the results. ${ }^{25}$ The weight-adjusted dose correction for gadolinium is not clearcut in the very obese population, but we elected to follow published methods. ${ }^{25}$ This resulted in subjects who lost weight receiving a lower dose of gadolinium at follow-up which may have influenced the results. However, the beneficial effects of weight loss were also evident in the cartilage thickness parameters which were independent of gadolinium dosing. In addition, the lack of change in the lateral dGEMRIC index suggests that the change in the medial compartment is due to a large extent to matrix improvement and is not dose-related. Recent body composition analysis in obese subjects after bariatric surgery showed that extracellular water does not change with weight loss. ${ }^{48}$ As GdDTPA ${ }^{2-}$ is distributed solely in extracellular water, ${ }^{13}$ this should not affect the dGEMRIC results. Future research incorporating body composition analysis (adipose tissue vs lean tissue) would be informative to interpret weight loss-mediated changes in cartilage.

In this cohort there was no association between baseline or change in alignment and subsequent cartilage loss. Varus-valgus alignment has been associated with subsequent progression of knee OA. ${ }^{49}$ However, most subjects in this cohort had varus alignment with little absolute change over 12 months, potentially reducing the likelihood of detecting any associations.

Improved knee range of motion was associated with reduced loss of cartilage thickness after adjustment for confounders. An association with reduced knee circumference was also detected. We had previously demonstrated an association between reduced range of knee movement and cartilage defect scores. ${ }^{23}$ Cartilage integrity is dependent on cyclical loading. ${ }^{50-53}$ Biomechanical and metabolic factors such as leptin and other adipocytokines are postulated to play a role in obesity-mediated OA. ${ }^{54}$ The mechanism behind weight loss-mediated improvements in the dGEMRIC index and reduced cartilage loss is probably due to a combination of these factors. ${ }^{556}$

In this non-randomised study, the group that underwent surgery had less cartilage thickness loss and larger improved dGEMRIC measures than the non-surgical group (data not shown). These differences are probably secondary to the higher weight loss observed in the surgical cohort. Potential biochemical and 
metabolic differences between the surgical and non-surgical groups (such as differences in absorption and cytokine levels) may in part also explain the differences, independent of weight loss. Randomised controlled trials comparing surgery and nonsurgical interventions will be necessary to assess the influence of these biochemical and metabolic differences on knee structural outcomes, but this will be difficult to achieve in practice as marked mean differences in weight loss between these two interventions are likely to remain.

In summary, this is the first prospective study to assess the impact of weight loss on important cartilage morphometric measures such as cartilage thickness and dGEMRIC index. This has important ramifications as our findings indicate that weight loss can lead to improved cartilage structural outcomes and may reduce the need for total joint replacements. This is pertinent as, to date, there are no drug therapies that can slow down cartilage loss. This has significant public health and economic implications, given the rising burden of both obesity and knee OA. ${ }^{57}$

Acknowledgements The authors thank Elisia Manson, Metabolism and Obesity Services, RPAH, and Sarah Fisher, NS Private Hospital, for help with accessing patient records; Peter Stanwell and Jeff McIntosh for performing the MR imaging; the Northern Clinical School, University of Sydney Advanced Research and Clinical High-field Imaging for management of the 3T facility; J S Chen, Rheumatology, RNSH, for statistical advice; Dr Felix Eckstein and Dr Martin Hudelmaier (Chondrometrics, Ainring, Germany) for guidance with the use of proprietary software for cartilage morphometric assessment; Professor Deborah Burstein, Department of Radiology, Beth Israel Deaconess Medical Center, Boston, Massachusetts, for her expert advice with dGEMRIC assessment; and the study participants.

Funding AA was supported by a National Health and Medical Research Council Medical Postgraduate Research Scholarship (402901).

Competing interests None.

Ethics approval This study was conducted with the approval of the Northern Sydney Central Coast Area Health Service Human Research Ethics Committee and University of Sydney and informed consent was obtained from all study participants.

Provenance and peer review Not commissioned; externally peer reviewed.

Contributors All authors contributed to the manuscript.

\section{REFERENCES}

1. World Health Organization. Global Strategy on Diet, Physical Activity, and Health. 2006. http://www.who.int/dietphysicalactivity/publications/facts/obesity/en/ (accessed 10 February 2011).

2. McTigue K, Larson JC, Valoski A, et al. Mortality and cardiac and vascular outcomes in extremely obese women. JAMA 2006;296:79-86.

3. Felson DT, Anderson JJ, Naimark A, et al. Obesity and knee osteoarthritis. The Framingham Study. Ann Intern Med 1988;109:18-24.

4. Hart DJ, Doyle DV, Spector TD. Incidence and risk factors for radiographic knee osteoarthritis in middle-aged women: the Chingford Study. Arthritis Rheum 1999;42:17-24.

5. Belo JN, Berger MY, Reijman M, et al. Prognostic factors of progression of osteoarthritis of the knee: a systematic review of observational studies. Arthritis Rheum 2007:57:13-26.

6. Nutristrategy. Costs of Obesity and Overweight. 2005. http://www.nutristrategy.com/ econcost.htm (accessed 10 February 2011).

7. National Collaborating Centre for Chronic Conditions. Osteoarthritis: National Clinical Guideline for Care and Management in Adults. London: Royal College of Physicians, 2008.

8. West R. Obesity. Office of Health Economics, 1994.

9. Cicuttini FM, Wluka AE, Forbes $A$, et al. Comparison of tibial cartilage volume and radiologic grade of the tibiofemoral joint. Arthritis Rheum 2003;48:682-8.

10. Huber M, Trattnig S, Lintner F. Anatomy, biochemistry, and physiology of articular cartilage. Invest Radiol 2000;35:573-80.

11. Anandacoomarasamy A, Giuffre BM, Leibman S, et al. Delayed gadoliniumenhanced magnetic resonance imaging of cartilage: clinical associations in obese adults. J Rheumatol 2009;36:1056-62.

12. Guermazi A, Burstein D, Conaghan P, et al. Imaging in osteoarthritis. Rheum Dis Clin North Am 2008;34:645-87.

13. Burstein D, Velyvis J, Scott KT, et al. Protocol issues for delayed Gd(DTPA)(2-)enhanced MRI (dGEMRIC) for clinical evaluation of articular cartilage. Magn Reson Med 2001;45:36-41.
14. Bashir A, Gray ML, Boutin RD, et al. Glycosaminoglycan in articular cartilage: in vivo assessment with delayed Gd(DTPA)(2-)-enhanced MR imaging. Radiology 1997;205:551-8.

15. Williams A, Gillis A, McKenzie C, et al. Glycosaminoglycan distribution in cartilage as determined by delayed gadolinium-enhanced MRI of cartilage (dGEMRIC): potential clinical applications. AJR Am J Roentgenol 2004;182:167-72.

16. Ericsson YB, Tjörnstrand J, Tiderius CJ, et al. Relationship between cartilage glycosaminoglycan content (assessed with dGEMRIC) and OA risk factors in meniscectomized patients. Osteoarthr Cartil 2009;17:565-70.

17. Messier SP, Loeser RF, Mitchell MN, et al. Exercise and weight loss in obese older adults with knee osteoarthritis: a preliminary study. J Am Geriatr Soc 2000;48:1062-72.

18. Huang $\mathbf{M H}$, Chen $\mathrm{CH}$, Chen TW, et al. The effects of weight reduction on the rehabilitation of patients with knee osteoarthritis and obesity. Arthritis Care Res 2000;13:398-405.

19. Altman R, Asch E, Bloch D, et al. Development of criteria for the classification and reporting of osteoarthritis. Classification of osteoarthritis of the knee. Diagnostic and Therapeutic Criteria Committee of the American Rheumatism Association. Arthritis Rheum 1986;29:1039-49.

20. Kraus VB, Vail TP, Worrell T, et al. A comparative assessment of alignment angle of the knee by radiographic and physical examination methods. Arthritis Rheum 2005:52:1730-5

21. Liikavainio T, Lyytinen T, Tyrväinen E, et al. Physical function and properties of quadriceps femoris muscle in men with knee osteoarthritis. Arch Phys Med Rehabil 2008;89:2185-94.

22. Williams A, Sharma L, McKenzie CA, et al. Delayed gadolinium-enhanced magnetic resonance imaging of cartilage in knee osteoarthritis: findings at different radiographic stages of disease and relationship to malalignment. Arthritis Rheum 2005;52:3528-35.

23. Anandacoomarasamy A, Smith G, Leibman S, et al. Cartilage defects are associated with physical disability in obese adults. Rheumatology (Oxford) 2009;48:1290-3.

24. Eckstein F, Charles HC, Buck RJ, et al. Accuracy and precision of quantitative assessment of cartilage morphology by magnetic resonance imaging at 3.0T. Arthritis Rheum 2005:52:3132-6.

25. Tiderius C, Hori M, Williams A, et al. dGEMRIC as a function of BMI. Osteoarthr Cartil 2006;14:1091-7.

26. Shai I, Schwarzfuchs D, Henkin Y, et al. Weight loss with a low-carbohydrate, Mediterranean, or low-fat diet. N Engl J Med 2008;359:229-41.

27. Church TS, Martin CK, Thompson AM, et al. Changes in weight, waist circumference and compensatory responses with different doses of exercise among sedentary, overweight postmenopausal women. PLoS ONE 2009;4:e4515.

28. Watkins BM, Ahroni JH, Michaelson R, et al. Laparoscopic adjustable gastric banding in an ambulatory surgery center. Surg Obes Relat Dis 2008;4(3 Suppl):S56-62.

29. Eckstein F, Maschek S, Wirth W, et al. One year change of knee cartilage morphology in the first release of participants from the Osteoarthritis Initiative progression subcohort: association with sex, body mass index, symptoms and radiographic osteoarthritis status. Ann Rheum Dis 2009;68:674-9.

30. Wirth W, Hellio Le Graverand MP, Wyman BT, et al. Regional analysis of femorotibial cartilage loss in a subsample from the 0steoarthritis Initiative progression subcohort. Osteoarthr Cartil 2009;17:291-7.

31. Eckstein F, Wirth W, Hudelmaier Ml, et al. Relationship of compartment-specific structural knee status at baseline with change in cartilage morphology: a prospective observational study using data from the osteoarthritis initiative. Arthritis Res Ther 2009;11:R90.

32. Hunter DJ, Niu J, Zhang Y, et al. Change in cartilage morphometry: a sample of the progression cohort of the Osteoarthritis Initiative. Ann Rheum Dis 2009;68:349-56.

33. Conaghan P. Is MRI useful in osteoarthritis? Best Pract Res Clin Rheumatol 2006;20:57-68

34. Williams TG, Holmes AP, Bowes M, et al. Measurement and visualisation of focal cartilage thickness change by MRI in a study of knee osteoarthritis using a novel image analysis tool. Br J Radiol 2010;83:940-8.

35. Le Graverand MP, Buck RJ, Wyman BT, et al. Change in regional cartilage morphology and joint space width in osteoarthritis participants versus healthy controls: a multicentre study using 3.0 Tesla MRI and Lyon-Schuss radiography. Ann Rheum Dis 2010;69:155-62.

36. Mündermann A, Dyrby CO, D'Lima DD, et al. In vivo knee loading characteristics during activities of daily living as measured by an instrumented total knee replacement. J Orthop Res 2008;26:1167-72.

37. Eckstein F, Cicuttini F, Raynauld JP, et al. Magnetic resonance imaging (MRI) of articular cartilage in knee osteoarthritis (OA): morphological assessment. Osteoarthr Cartil 2006;14(Suppl A):A46-75.

38. Eckstein F, Gavazzeni A, Sittek H, et al. Determination of knee joint cartilage thickness using three-dimensional magnetic resonance chondro-crassometry (3D MR-CCM). Magn Reson Med 1996;36:256-65

39. Marshall KW, Guthrie BT, Mikulis DJ. Quantitative cartilage imaging. Br J Rheumatol 1995;34(Suppl 1):29-31.

40. Wluka AE, Forbes A, Wang Y, et al. Knee cartilage loss in symptomatic knee osteoarthritis over 4.5 years. Arthritis Res Ther 2006;8:R90. 
41. Cicuttini FM, Jones $\mathrm{G}$, Forbes $\mathrm{A}$, et al. Rate of cartilage loss at two years predicts subsequent total knee arthroplasty: a prospective study. Ann Rheum Dis 2004;63:1124-7.

42. Wluka AE, Ding C, Jones G, et al. The clinical correlates of articular cartilage defects in symptomatic knee osteoarthritis: a prospective study. Rheumatology (Oxford) 2005; $44: 1311-16$

43. Owman H, Tiderius CJ, Neuman P, et al. Association between findings on delayed gadolinium-enhanced magnetic resonance imaging of cartilage and future knee osteoarthritis. Arthritis Rheum 2008;58:1727-30.

44. Young AA, Stanwell P, Williams A, et al. Glycosaminoglycan content of knee cartilage following posterior cruciate ligament rupture demonstrated by delayed gadoliniumenhanced magnetic resonance imaging of cartilage (dGEMRIC). A case report. J Bone Joint Surg Am 2005;87:2763-7.

45. Tiderius CJ, Olsson LE, Nyquist F, et al. Cartilage glycosaminoglycan loss in the acute phase after an anterior cruciate ligament injury: delayed gadolinium-enhanced magnetic resonance imaging of cartilage and synovial fluid analysis. Arthritis Rheum 2005;52:120-7.

46. Tiderius $\mathbf{C J}$, Olsson LE, Leander $\mathrm{P}$, et al. Delayed gadolinium-enhanced $\mathrm{MRI}$ of cartilage (dGEMRIC) in early knee osteoarthritis. Magn Reson Med 2003;49:488-92.

47. Gray ML, Burstein D, Kim YJ, et al. 2007 Elizabeth Winston Lanier Award Winner. Magnetic resonance imaging of cartilage glycosaminoglycan: basic principles, imaging technique, and clinical applications. J Orthop Res 2008:26:281-91.

48. Levitt DG, Beckman LM, Mager JR, et al. Comparison of DXA and water measurements of body fat following gastric bypass surgery and a physiological model of body water, fat, and muscle composition. J Appl Physiol 2010;109:786-95.
49. Moisio K, Chang A, Eckstein F, et al. Varus-valgus alignment: reduced risk of subsequent cartilage loss in the less loaded compartment. Arthritis Rheum 2011;63:1002-9.

50. Vanwanseele B, Lucchinetti E, Stüssi E. The effects of immobilization on the characteristics of articular cartilage: current concepts and future directions. Osteoarthr Cartil 2002;10:408-19.

51. Arokoski JP, Jurvelin JS, Väätäinen U, et al. Normal and pathological adaptations of articular cartilage to joint loading. Scand J Med Sci Sports 2000;10:186-98.

52. Radin EL, Burr DB, Caterson B, et al. Mechanical determinants of osteoarthrosis. Semin Arthritis Rheum 1991:21 (3 Suppl 2):12-21.

53. Buckwalter JA. Osteoarthritis and articular cartilage use, disuse, and abuse: experimental studies. J Rheumatol Supp/ 1995;43:13-15.

54. Anandacoomarasamy A, Fransen M, March L. Obesity and the musculoskeletal system. Curr Opin Rheumatol 2009;21:71-7.

55. Miller GD, Nicklas BJ, Loeser RF. Inflammatory biomarkers and physical function in older, obese adults with knee pain and self-reported osteoarthritis after intensive weight-loss therapy. J Am Geriatr Soc 2008;56:644-51.

56. Miller GD, Nicklas BJ, Davis CC, et al. Is serum leptin related to physical function and is it modifiable through weight loss and exercise in older adults with knee osteoarthritis? Int J Obes Relat Metab Disord 2004;28:1383-90.

57. Anandacoomarasamy A, Caterson I, Sambrook P, et al. The impact of obesity on the musculoskeletal system. Int J Obes (Lond) 2008;32:211-22.

58. Eckstein F, Ateshian G, Burgkart R, et al. Proposal for a nomenclature for magnetic resonance imaging based measures of articular cartilage in osteoarthritis. Osteoarthr Cartil 2006:14:974-83 